\title{
Bias- and bath-mediated pairing of particles driven through a quiescent medium
}

\author{
Carlos Mejía-Monasterio $^{* a b}$ and Gleb Oshanin ${ }^{c d}$
}

\author{
Received 2nd June 2010, Accepted 13th October 2010 \\ DOI: 10.1039/c0sm00465k
}

A particle driven by an external force in a molecular crowding environment - a quiescent bath of other particles, makes their spatial distribution inhomogeneous: the bath particles accumulate in front of the biased particle (BP) and are depleted behind. In fact, a BP travels together with the inhomogeneity it creates. A natural question is what will happen with two BPs when they appear sufficiently close to each other such that the inhomogeneities around each of them start to interfere? In quest for the answer we examine here, via Monte Carlo simulations, the dynamics of two BPs in a lattice gas of bath particles. We observe that for a sufficiently dense medium, surprisingly, both BPs spend most of the time together which signifies that the interference of the microstructural inhomogeneities results in effectively attractive interactions between them. Such statistical pairing of BPs minimizes the size of the inhomogeneity and hence reduces the frictional drag force exerted on the BPs by the medium. As a result, in some configurations the center-of-mass of a pair of BPs propagates faster than a single isolated BP. These jamming-induced forces are very different from fundamental physical interactions, exist only in presence of an external force, and require the presence of a quiescent bath to mediate the interactions between the driven particles.

\section{Introduction}

A biased particle (BP) travelling in a bath of particles, which move randomly without any preferential direction, drives their spatial distribution out of equilibrium. The bath particles accumulate, creating a "traffic jam" in front of the BP and are depleted behind it. This BP can be, e.g., a charge carrier subject to an electric field or a colloid moved with an optical tweezer. The bath particle may be, e.g., colloids dispersed in a solvent or adatoms performing activated hopping motion among the adsorption sites on a solid surface.

Such microstructural changes, which substantially enhance the drag force exerted on the BP, have been observed experimentally; in particular, in microrheological measurements of the drag force on a single colloid driven through a $\lambda$-DNA solution $^{1}$ or for a biased motion of an intruder dragged into a monolayer of vibrated grains. ${ }^{2}$ Formation of an inhomogeneous nonequilibrium distribution has also been revealed by Brownian Dynamics simulations of a driven colloid in a $\lambda$-DNA solution ${ }^{1,3}$ and in a colloidal crystal. ${ }^{4}$ In the latter case, it was shown that a large enough BP generates a sufficient stress to produce defects, which remain localized near the $\mathrm{BP}$ and affect the frictional drag force.

${ }^{a}$ Department of Mathematics and Statistics, University of Helsinki, P.O. Box 68, FIN-00014 Helsinki, Finland

${ }^{b}$ Laboratory of Physical Properties, Department of Rural Engineering, Technical University of Madrid, Av. Complutense s/n, 28040 Madrid, Spain.E-mail: carlos.mejia@upm.es

'Laboratoire de Physique Théorique de la Matière Condensée (UMR CNRS 7600), Université Pierre et Marie Curie, 4 place Jussieu, 75252 Paris Cedex 5, France

${ }^{d}$ Laboratory J.-V. Poncelet (UMI CNRS 2615), Independent University of Moscow, Bolshoy Vlasyevskiy Pereulok 11, 119002 Moscow, Russia
Microstructural changes of a quiescent medium caused by a biased probe were extensively studied analytically ${ }^{5-11}$ for hardcore lattice gases with simple exclusion dynamics, in which all particles except one have symmetric hopping probabilities, while one of them - the BP — has a preferential direction of motion.

In one-dimensional systems the size of the jammed region in front of a BP (as well as the size of the depleted region in the wake) grows in proportion to the travelled distance. Thus the jamming-induced contribution to the frictional drag force $\gamma$ exerted on the BP by the bath particles exhibits an unbounded growth, $\gamma \approx t^{1 / 2}$, ( $t$ being time $)$, so that the BP velocity $V_{t}^{(1)}$ vanishes, ${ }^{5,6} V_{t}^{(1)} \approx t^{-1 / 2}$ as $t \rightarrow \infty$. This insures the validity of the Einstein relation for anomalous diffusion in one-dimensional hard-core lattice gases. ${ }^{6,7}$

In higher dimensions, the BP velocity attains a drift value $V^{(1)}=V_{t=\infty}^{(1)}$ and the bath particle distribution reaches a nonequilibrium stationary form. ${ }^{8-11}$ The density profiles are strongly anisotropic with a traffic-jam like region in front of the BP and a depleted region in its wake. Strikingly, behind the BP the bath particle density approaches the mean value $\rho$ as a power-law of the distance $x: 1 / x^{3 / 2}$ and $\ln (x) / x^{2}$ in $2 \mathrm{D}$ and $3 \mathrm{D}$, respectively, ${ }^{8-11}$ which signifies that the medium "remembers" the passage of the BP on large temporal and spatial scales. The drift velocity $V^{(1)}$ and the jamming-induced drag force $\gamma$ have been determined for the lattice gas model $^{7-11}$ and also for a driven probe in a colloidal mixture. . $^{12,13}$

The next step in the understanding of the jamming-induced forces has been done in Ref. 14-16, Dzubiella et al. ${ }^{14}$ studied the effective interactions between two fixed colloids in a quiescent viscous solvent exposed to a flowing bath of small Brownian particles, while Krüger and Rauscher ${ }^{15}$ and Khair and Brady ${ }^{16}$ considered the case of two colloids translating along their lines of centres with fixed velocities and a fixed distance apart from each 
other in an otherwise quiescent dispersion of noninteracting colloids. It was realized that, remarkably, microstructural changes induce effective forces between two colloids, which may be either repulsive or attractive, depending on their mutual orientation.

In this paper we pose a very natural question within the context of microfluidics/microrheology or biased dynamics under molecular crowding conditions: what will happen with two BPs in a quiescent medium of mutually interacting particles when both move, not with a prescribed velocity along some fixed lines, but rather perform a biased random motion subject to some external force? In contrast to the situations studied in Ref. 3, 14 and 16 , here the BPs can change their relative position in space and hence, by monitoring their trajectories we can understand the overall effect of microstructural changes of the medium on the interactions between them.

In order to be as transparent as possible, here we resort to a minimal model of a hard-core lattice gas of particles whose dynamics obeys the so-called simple exclusion process (SEP). ${ }^{17,18}$ We note parenthetically that this model of dynamics is quite realistic and applies to many physical systems, such as, e.g., dynamics of adatoms on solid surfaces (see Ref. 8-11, 17 and 18 for more details and other systems). We note, as well, that it allows us to single out the effect of microstructural changes, i.e., bath-mediated interactions, and to exclude possible effects of solvent (if present) and solvent-mediated interactions ${ }^{19}$ between the BPs.

In this model the lattice gas particles - the bath particleshave symmetric hopping probabilities while two particles - the BPs - are subject to an external force and have asymmetric hopping probabilities. Tracking the BPs' trajectories in Monte Carlo simulations, we observe a phenomenon of statistical pairing of biased particles. We realize that for sufficiently dense systems the fraction of time which the second BP spends at a given point in space has an apparent maximum in the vicinity of the first BP. Hence, the interference of non-equilibrium density profiles of the bath particles formed around each of the BPs results in an effective attractive interaction between them. Apart from this, we also analyze the properties of the jamming-induced frictional forces and determine the velocities of the BPs appearing in different configurations.

The paper is outlined as follows: in Section 2 we define the model. In Section 3 we focus on dynamics of a single biased particle in a quiescent bath, describe the density profiles of the bath particles around the BP and determine the force-velocity relation. In Section 4 we consider dynamics of two BPs. Here we define the most probable paths that the BPs follow and describe the density profiles of the bath particles forming around a pair of BPs appearing in different configurations. Apart from this, we also define the frictional force exerted by the medium on the BPs and determine the velocity of a pair of BPs. We conclude in Section 5 with a brief recapitulation of our results and an outlook of future work.

\section{The model}

Consider a square lattice of $S \equiv L_{x} \times L_{y}$ sites, of spacing $\sigma$ and with periodic boundary conditions. The lattice is populated with two different types of particles: $N-M$ bath particles and

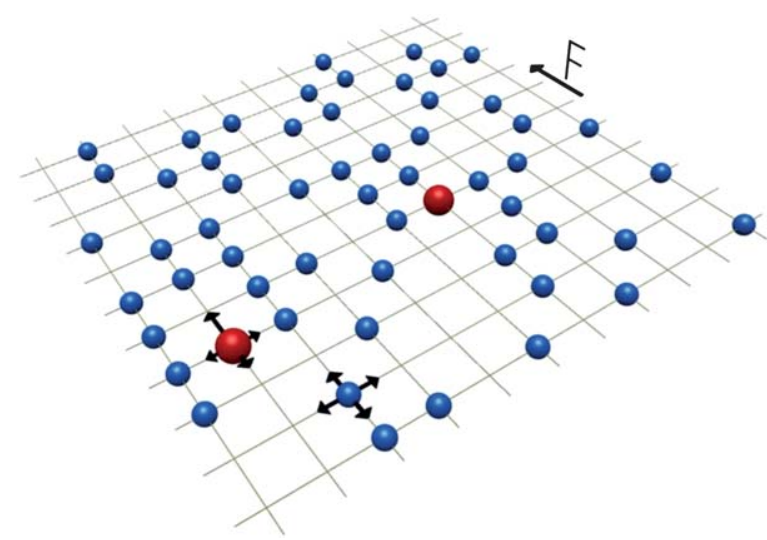

Fig. 1 A lattice with randomly moving hard-core particles. Bath (blue) particles have symmetric hopping probabilities. BPs (red) are subject to a constant force and have asymmetric hopping probabilities.

$M(M=1,2)$ BPs (see Fig. 1). All particles experience hard-core interactions, such that each site can be either empty or occupied by at most one particle. The state of each site $(X, Y)$ is described by a time-dependent occupation variable $\eta(X, Y) ; \eta(X, Y)=1$ if the site $(X, Y)$ is occupied and $\eta(X, Y)=0$, otherwise.

Particle dynamics is defined by the so-called SEP-simple exclusion process. ${ }^{17,18}$ Each particle bears an exponential clock; in general, the mean jump-time of the bath particles and that of the BPs may be different; say, it is $\tau^{*}$ for the bath particles, while for the BPs it is $\tau$. We will focus in what follows on the simplest case $\tau^{*}=\tau=1$ and will only briefly mention the effects of different jump times on the friction coefficient.

When the clock rings, a particle attempts to jump from the site $(X, Y)$ it occupies to one of the four nearest-neighboring sites $\left(X^{\prime}, Y^{\prime}\right)$ according to the normalized set of probabilities $p\left(X, Y \mid X^{\prime}, Y^{\prime}\right)$. Once a jump direction is chosen, and if the destination site is empty, the particle moves to it, otherwise it remains on the site it occupies. This stochastic exclusion dynamics is a Markov process on a state space

$$
\mathscr{M}=\{0,1\}^{L_{x}} \otimes\{0,1\}^{L_{y}}
$$

We stipulate next that the dynamics of the bath particles obey a symmetric SEP (all hopping probabilities $=1 / 4$ ), while the BPs are driven by an external field $\vec{F}=-F \hat{e}_{X}$ and evolve according to an asymmetric SEP:

$$
p\left(\mathbf{r}, \mathbf{r} \pm \sigma \hat{\mathbf{e}}_{X}\right)=Z^{-1} e^{\mp \beta \sigma F / 2}, p\left(\mathbf{r}, \mathbf{r} \pm \sigma \hat{\mathbf{e}}_{Y}\right)=Z^{-1},
$$

where $\hat{\mathbf{e}}_{X}=(1,0)$ and $\hat{\mathbf{e}}_{Y}=(0,1)$ are unit shift vectors, $\mathbf{r}=(X, Y)$, $Z=2(1+\cosh (\beta \sigma F / 2))$ and $\beta$ is the inverse temperature.

\section{One biased particle}

To set up the scene, we focus first on the case of a single BP on a lattice with $N-1$ bath particles. As we have already remarked, a single BP produces microstructural changes in the medium it travels in, driving the spatial distribution of the bath particles out of equilibrium. To quantify the microstructural changes of the medium, we consider the following realization-dependent "inhomogeneity" measure: 


$$
g_{0}(\mathbf{r} ; t)=\frac{1}{t} \int_{0}^{t} d \tau\left(\frac{1}{\rho} \sum_{i=2}^{N} \delta\left(\mathbf{r}_{i}(\tau)-\mathbf{R}_{1}(\tau)-\mathbf{r}\right)\right),
$$

where $\delta(\cdot)$ is the Kronecker-delta, which equals 1 when its argument is 0 and is zero otherwise, $\rho=(N-1) /(S-1)$ is the mean density of bath particles, while $\mathbf{R}_{1}(\tau)$ and $\mathbf{r}_{i}(\tau)$ denote the positions of the BP and of the $i$-th bath particle at time $\tau$, respectively, for a given set of realizations of their trajectories. An average of $g_{0}(\mathbf{r} ; t)$ over different realizations of particles' trajectories defines the time-averaged van Hove function. ${ }^{20}$

The realization-dependent functional $g_{0}(\mathbf{r} ; t)$ in eqn (2) defines the fraction of time the site $\mathbf{r}=(x, y)$, in the frame of reference moving with the $\mathrm{BP}$, is occupied by bath particles during a time $t$ for a given realization of trajectories of the bath particles and the $\mathrm{BP} . \dagger$ If the spatial distribution of the bath particles converges to a stationary form, i.e., if $\lim _{t \rightarrow \infty} g_{0}(\mathbf{r} ; t)=g_{0}(\mathbf{r})$ exists, then $\rho g_{0}(\mathbf{r})$ can also be thought of as the bath particle's density profile as seen from a stationary moving BP. Clearly, $g_{0}(\mathbf{0})=0$ due to the hardcore exclusion and $g_{0}(\mathbf{r}) \rightarrow 1$ when $|\mathbf{r}| \rightarrow \infty$. We have conveniently normalized $g_{0}(\mathbf{r} ; t)$ to $\rho$, so that any deviation $g_{0} \neq 1$ indicates a non zero dynamical correlation between the BP and the medium.

In Fig. 2 we depict the bath particles density $g_{0}(\mathbf{r})$ using a colour map. The density profiles around a stationary moving $\mathrm{BP}$ are characterized by a jammed, high-density region in front of the $\mathrm{BP}$ and a pronounced region depleted by the bath particles past the BP. This agrees quite well with the theoretical prediction of Ref. 7-10. We verify, as well, the theoretical prediction that the density past the BP approaches the average value $\rho$ not exponentially with the distance $x$, but as a slow power-law $x^{-3 / 2}$. Moreover, in Fig. 2 we superimpose the average velocity field, which shows that the BP induces a regular global motion of the bath particles predominantly towards the regions with lower

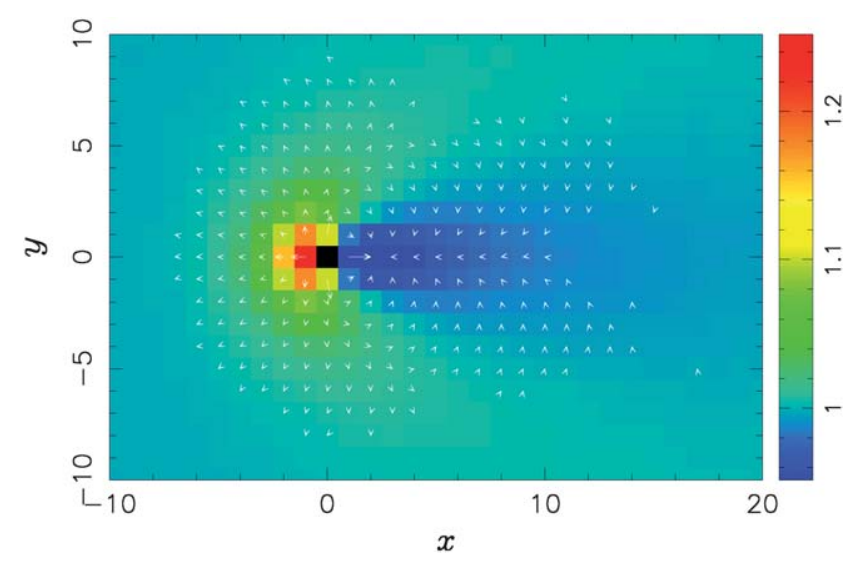

Fig. 2 Microstructural changes of the medium produced by a single BP. The profile $g_{0}(x, y)$ in eqn (2) is shown for a lattice comprising $61 \times 21$ sites at density $\rho=1 / 2$ and $\beta \sigma F=5$. The vector field shows the average velocity field of the bath particles defined in the reference frame of the BP (a black square). Velocity vectors with magnitude less than 0.0005 are not plotted.

$\dagger$ Here and henceforth, small characters $x$ and $y$ will denote a coordinate system moving with the BP, while $X$ and $Y$ will denote the laboratory frame of reference. density. Note that similar motion patterns have been observed experimentally for biased motion in granular media. ${ }^{2}$

Next, in Fig. 3 we depict the dependence of the BP's drift velocity $V^{(1)}$ on the applied force $F$, i.e. the force-velocity relation, for $\rho=1 / 2$ The notable feature of the observed force-velocity curve is that in the limit of sufficiently small forcing (sufficiently high temperatures) the drift velocity shows a Stokesian, linear dependence on the applied force, $V^{(1)}=F / \xi$, which signifies that in this limit the frictional force exerted by the bath particles on the BP is viscous.

According to Ref. 7-10 (see also Section 4 in Ref. 21), in this linear regime the friction coefficient $\xi$ can be expressed as a sum of two contributions,

$$
\xi=\xi_{\mathrm{mf}}+\xi_{\mathrm{coop}}
$$

where the first term,

$$
\xi_{\mathrm{mf}}=\frac{4 \tau}{\beta \sigma^{2}(1-\rho)},
$$

is essentially a mean-field result corresponding to a perfectly stirred monolayer; one may interpret $(1-\rho) / \tau$ just as the frequency of the BPs "successful" jump events. The second term is a "jamming-induced" contribution:

$$
\xi_{\text {coop }}=\frac{4 \tau^{*}}{\beta \sigma^{2}(1-\rho)} \frac{(\pi-2) \rho}{1+(1-\rho) \tau^{*} / \tau}
$$

stemming out of a cooperative behavior in the monolayera non-linear interplay between the BP dynamics and the formation of non-equilibrium density profiles around it. Analogous results for $\xi$ have been obtained for three-dimensional $^{11}$ and one-dimensional ${ }^{5,6}$ systems; in the latter case $\xi$ diverges as $t \rightarrow \infty$.

Dividing $\xi_{\mathrm{mf}}$ by $\xi_{\mathrm{coop}}$, we have

$$
\frac{\xi_{\mathrm{mf}}}{\xi_{\mathrm{coop}}} \sim \frac{1-\rho}{\rho}+\frac{1}{\rho} \frac{\tau}{\tau^{*}}
$$

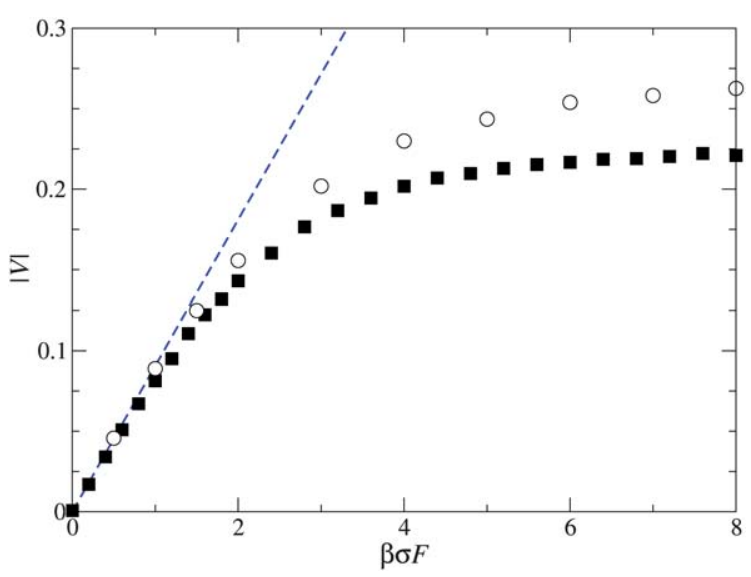

Fig. 3 Force-velocity relation. Drift velocity $V^{(1)}$ of a single BP $v s$. $F$ (solid squares). The dashed line is the theoretical prediction $V^{(1)}=F / \xi$ in which the friction coefficient $\xi$ is given by eqn (3). Open circles denote the results of the Monte Carlo simulations for the drift velocity of a pair of BPs in the $(2,0)$ configuration (see the explanations in Section 4.2). 
One notices that the jamming-induced contribution to the friction coefficient dominates when $\tau^{*} \gg \tau$ and the bath particles mean density is not too small. Conversely, the mean-field contribution is clearly the dominant one when $\rho \ll 1$ or $\tau^{*} \ll \tau$. For moderate densities, $\xi_{\mathrm{mf}}$ and $\xi_{\text {coop }}$ are comparable.

Finally, in Fig. 4 we compare the result in eqn (3) against Monte Carlo simulation results for the slope of the force-velocity relation in the limit of small forcing, at different densities $\rho$ and $\tau=\tau^{*}=1$. One observes a very good agreement between a theoretical prediction and numerical data.

\section{Two biased particles}

We turn now to the case of primary interest - two BPs. We suppose that initially the BPs are placed at sites $(X, Y)$ and $\left(X^{\prime}, Y^{\prime}\right)$, and that $X<X^{\prime}$; following the terminology of Khair and Brady, ${ }^{16}$ we then refer to the particle initially at $(X, Y)$ as the "leading" BP, and the one at $\left(X^{\prime}, Y^{\prime}\right)$ - the "trailing" BP (see Fig. 1). We recall that the coordinate system defined in the reference frame of the leading BP is denoted by $(x, y)$.

\subsection{Mutual orientation of the BPs}

We concentrate first on the analysis of the most probable paths of the trailing BP in the reference frame of the leading BP. For this purpose, we study numerically the behaviour of a realization-dependent functional

$$
g(\mathbf{r} ; t)=\frac{1}{t-t_{0}} \int_{t_{0}}^{t} d \tau \delta\left(\mathbf{R}_{2}(\tau)-\mathbf{R}_{1}(\tau)-\mathbf{r}\right),
$$

where $\mathbf{R}_{1}(\tau)$ and $\mathbf{R}_{2}(\tau)$ stand for the instantaneous positions of the leading and trailing particles, respectively, for a given realization of their trajectories. Similarly to the functional defined in eqn (2), $g(\mathbf{r} ; t)$ defines, for a given realization of the leading and trailing BPs trajectories, the fraction of time during the time interval $t-t_{0}$ that the site $\mathbf{r}$, (in the frame of reference moving with the leading BP), has been occupied by the trailing BP.

In simulations the leading BP is initially placed at the origin, the trailing one is placed at position $\mathbf{R}_{2}(0)$, while the bath particles are placed at random, with mean density $\rho$, on the rest

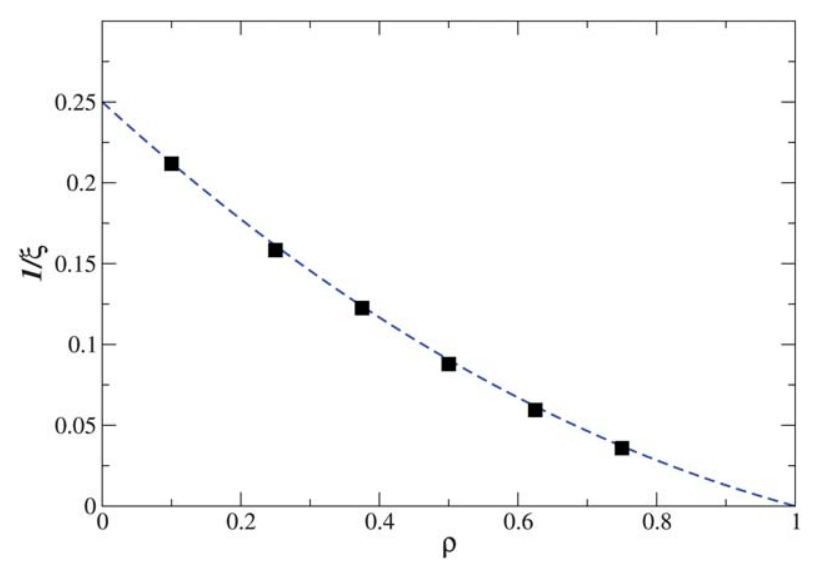

Fig. 4 Friction coefficient $\boldsymbol{\xi}$ versus density. The dashed line is the theoretical prediction in eqn (3), while the symbols define the numerical simulations results for $\sigma=\beta=1$ and $\tau=\tau^{*}=1$. of the lattice. We let the system evolve (the bath particles follow a standard symmetric SEP while the BPs' dynamics obeys an asymmetric SEP) for $10^{8}$ time steps, until the density profile of the bath particles around two BPs attains a stationary form. After this transient period, we define the moment $t_{0}$ when the trailing BP re-appears again at site $\mathbf{R}_{2}(0)$. Then, during the next $t-t_{0}=10^{6}$ time steps we evaluate $g(\mathbf{r} ; t)$ by recording the number of times each site $\mathbf{r}$ in the frame of reference of the leading BP has been visited by the trailing $\mathrm{BP}$ within this realization of the process.

In Fig. 5 we plot the average local occupation times $\langle g(\mathbf{r} ; t)\rangle \ddagger$ for $\beta \sigma F=5$ and $\mathbf{R}_{2}(0)=(3,4)$. Our results show that when the inhomogeneities around each BP do not overlap, both BPs travel almost independently. In this case, the profile of the average occupation times around the initial position of the trailing BP is almost symmetric (see Fig. 5(a)) with a small second maximum just after the leading BP. The overlap becomes significant for either sufficiently high density $\rho$, larger driving force $\beta \sigma F$ or naturally, when the leading and the trailing BPs are close enough. For progressively higher densities of the medium particles (see Fig. 5(b)-(d)) we observe a considerable qualitative change in the form of the profile $\langle g(\mathbf{r} ; t)\rangle$ : it becomes considerably more asymmetric and is characterized by an apparent "bridge" connecting the leading and the trailing BPs. Hence, the probability of finding the trailing $\mathrm{BP}$ in the vicinity of the leading one is getting progressively higher.

Further on, we focus on a single (very long) trajectory of a trailing BP (see Fig. 6). We let the system evolve for $10^{8}$ time steps to ensure that the bath particles distribution around the two BPs reaches a stationary form. Then, we evaluate $g(\mathbf{r} ; t)$ by tracking the trajectory of the trailing BP in the frame of reference of the leading one. We have checked that for sufficiently large times (in our simulations $\left.t=10^{8}\right), g(\mathbf{r} ; t)$ converges to a stationary function $g(\mathbf{r})$, which moreover, is independent of the initial state.

Fig. 6 shows the local occupation times $g(\mathbf{r} ; t)$ of a single trajectory together with the relative velocity field of the trailing BP with respect to the leading one. The circulating structure of this field indicates that the pair is statistically stable, presenting a stable direction ( $x$-axis) along which the trailing and the leading BPs "pair", and an unstable direction ( $y$-axis) along which the trailing BP moves away from the leader.

This signifies that when the density profiles of the bath particles emerging around each of the BPs start to interfere, the jamming-induced frictional force exerted on the trailing BP is no longer parallel to the $x$-axis (direction of the external force $F$ ) but is tilted by some angle pointing towards the wake of the leading BP. As a consequence, the trailing BP experiences an effective attraction towards the leading one such that both driven BPs statistically "pair". From the microscopic dynamics viewpoint, this interference modifies locally the statistics of successful hops of the trailing BP, increasing the likelihood of hops towards the wake of the leading BP.

To substantiate this claim, we have computed numerically an instantaneous jamming-induced nonequilibrium force $\vec{\gamma}(\mathbf{r} ; t)$ that we define as

\$ The angle brackets denote averaging over different realizations of the leading and trailing BPs trajectories. We consider $10^{3}$ such realizations. 

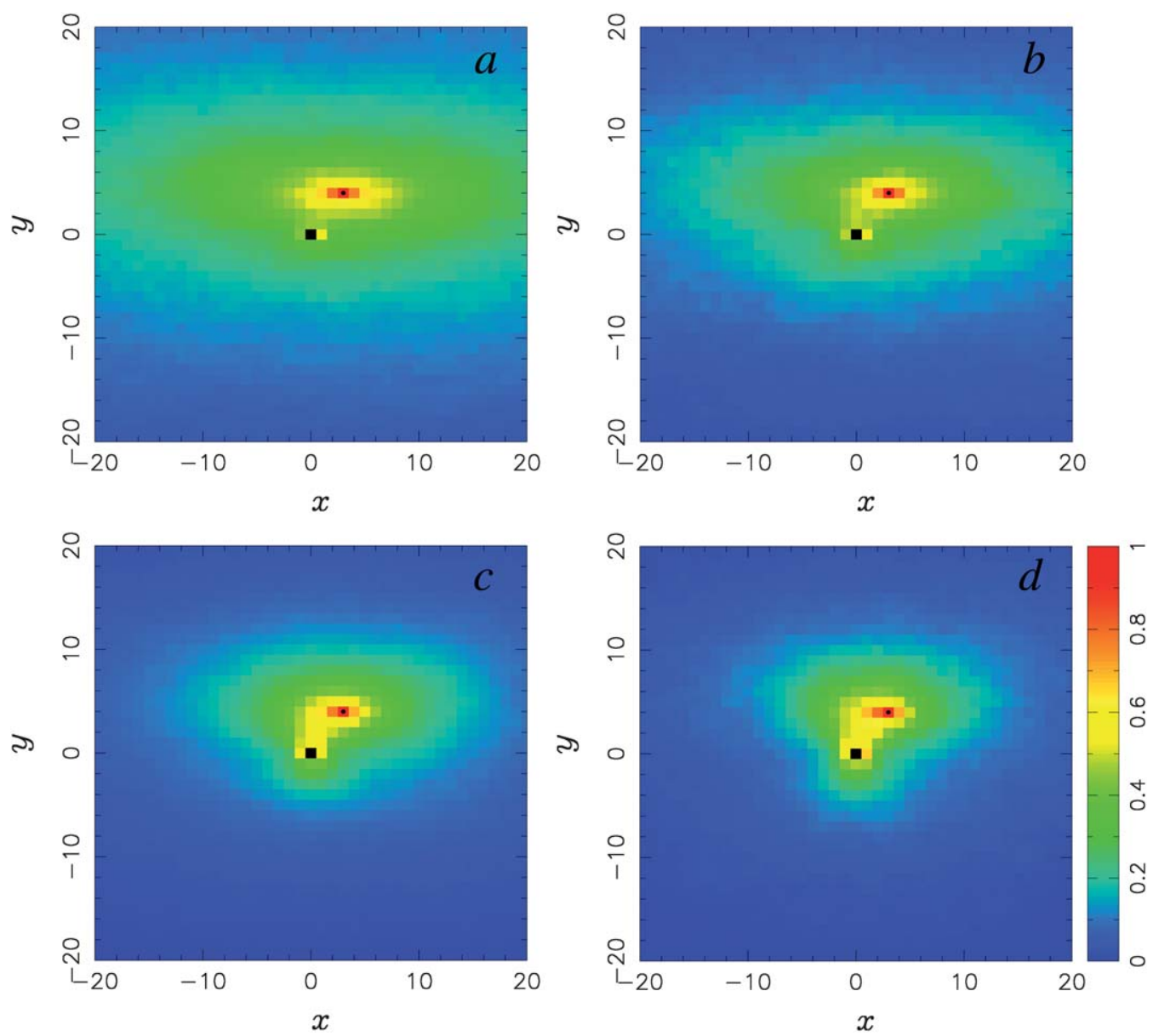

Fig. 5 Most probable paths. The profile of average local occupation times $\langle g(\mathbf{r} ; t)\rangle$, eqn (7), for the trailing BP commencing at $\mathbf{R}_{2}(0)=(3,4)($ little black knot), $\beta \sigma F=5$ and densities $\rho$ : (a) $1 / 4$, (b) $1 / 2$, (c) $3 / 4$ and (d) 0.9 . High values of $\langle g(\mathbf{r} ; t)\rangle$ (yellow) indicate the most probable paths that the trailing BP follows.

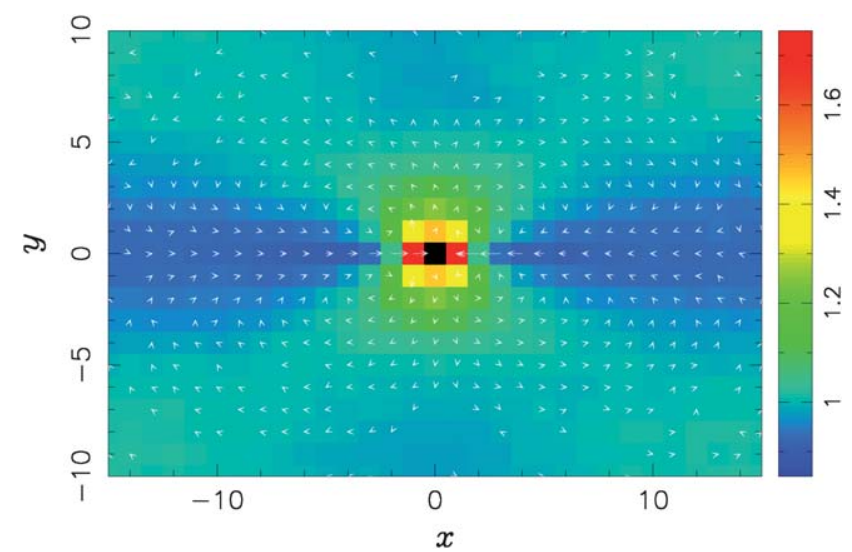

Fig. 6 Local occupation times of the trailing BP. Colour map of the occupation time of the trailing BP given by $g(\mathbf{r}) /(S-1)$, for a lattice comprising $31 \times 21$ sites, $\rho=1 / 2$ and $\beta \sigma F=5$. The superimposed vector field shows the average relative velocity field of the trailing BP measured in the reference frame of the leading BP. Velocity vectors with magnitude less than 0.001 are not plotted.

$$
\vec{\gamma}(\mathbf{r} ; t)=\sum_{i=\{x, y\}}\left(\eta\left(\mathbf{r}(\mathbf{t})-\hat{e}_{i}\right)-\eta\left(\mathbf{r}(\mathbf{t})+\hat{e}_{i}\right)\right) \hat{e}_{i}
$$

where $\eta(\mathbf{r}(\mathbf{t}))$ are the local occupation variables defined in Section 2. Clearly, $\vec{\gamma}(\mathbf{r} ; t)$ is the force that is felt by the trailing BP being at site $\mathbf{r}$ with respect to the leading BP at time moment $t$.

On average, $\vec{\gamma}(\mathbf{r})$ will be different from zero only if the distribution of the bath particles is inhomogeneous. In general, as follows from Fig. $6, \vec{\gamma}(\mathbf{r})$ should depend on the position of the trailing BP with respect to the leading one. In Fig. 7 we show the time averaged $y$ component of the force $\vec{\gamma}$ that the trailing BP experiences when it is at position $(0,7)$ (circles) and $(4,2)$ (squares) with respect to the leader. In the first case, the trailing BP is far enough from the leader so that the net force along $y$ is numerically zero, irrespective of the strength of the field. On the contrary, in the second case, the trailing BP is in the stable basin of attraction and $\gamma_{y}$ is negative, indicating that (in agreement with Fig. 6), the force pushes the trailing BP towards the leading one. For small fields $\gamma_{y}$ grows linearly with $\beta \sigma F$ and saturates at larger fields. Of course, the amplitude of the force $\vec{\gamma}(\mathbf{r})$ depends on the density of bath particles, external force $F$ and, naturally, on the temperature, which controls the rate of the bath particles' migration and thus their capability for smoothing down the inhomogeneities created by the BPs. 


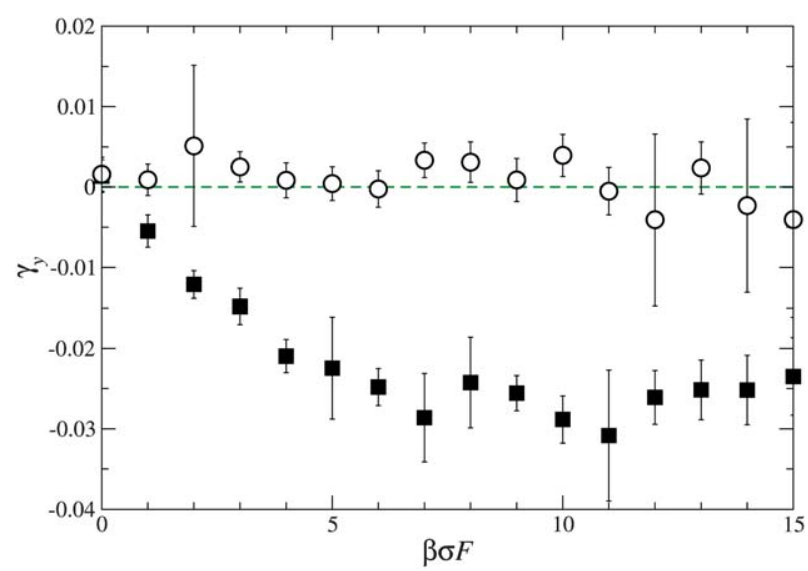

Fig. 7 Force exerted on the trailing BP. Time averaged jamming-induced force experienced by the trailing BP at position $(0,7)$ (open circles) and $(4,2)$ (solid squares), in the reference frame of the leading one, as a function of $\beta \sigma F$. The error bars indicate the standard deviation.

\subsection{The BPs velocities}

The (mean) drift velocity $V^{(1)}$ of a single isolated BP is totally determined by $\beta \sigma F, \rho$ and the rate of the bath particles' migration. ${ }^{9,10}$ For two BPs, when they appear sufficiently close to each other such that the inhomogeneities around each of them start to interfere, their drift velocities and the velocity of their center of mass will also depend on their mutual orientation. One may expect that only at large mutual separations of the BPs their velocities are equal to $V^{(1)}$.
To clarify this issue, we have studied the velocities of the leading and trailing BPs at a fixed force $\beta \sigma F=5$ and at a fixed bath-particle density $\rho=1 / 2$, but for different mutual orientations. Six different mutual orientations of the BPs, together with the corresponding microstructural changes of the medium, are presented in Fig. 8. In this figure we also depict the velocity field of the bath particles.

Further on, in Fig. 9 we plot the instantaneous drift velocities of the leading and trailing BPs as the function of their mutual orientation. In the left panels, along the horizontal line, each "tick" corresponds to the position $(x, y)$ of the trailing BP in the reference frame of the leading one. Between each pair of vertical dotted lines, we place nine points with fixed $y$ and $x$ varying from 1 to 9 , i.e., $(1, y),(2, y)$ to $(9, y)$. From the left lower panel one indeed concludes that at large separations the velocities of the BPs are nearly the same, and coincide with the velocity $V^{(1)}$ (blue dashed line) of a single isolated BP. Conversely, when the BPs are close enough, their velocities may be very different from each other, as well as from $V^{(1)}$.

At a fixed $y$, the velocity of the trailing BP is always a nonmonotonic function of its $x$-coordinate: it is always minimal for $x=0$, grows abruptly with the $x$-coordinate and then decays towards $V^{(1)}$. The leading BP velocity, at a fixed $y$-coordinate of the trailing BP, is a growing function of the $x$-coordinate of the latter. Despite the fact that the configuration $(1,0)$ is the most probable (see Fig. 6), the velocity of the center of mass (solid blue line in Fig. 9) of such a pair is not the largest one, which is a bit counter intuitive. As a matter of fact, this is the consequence of the hard-core interaction between the leading and trailing BPs
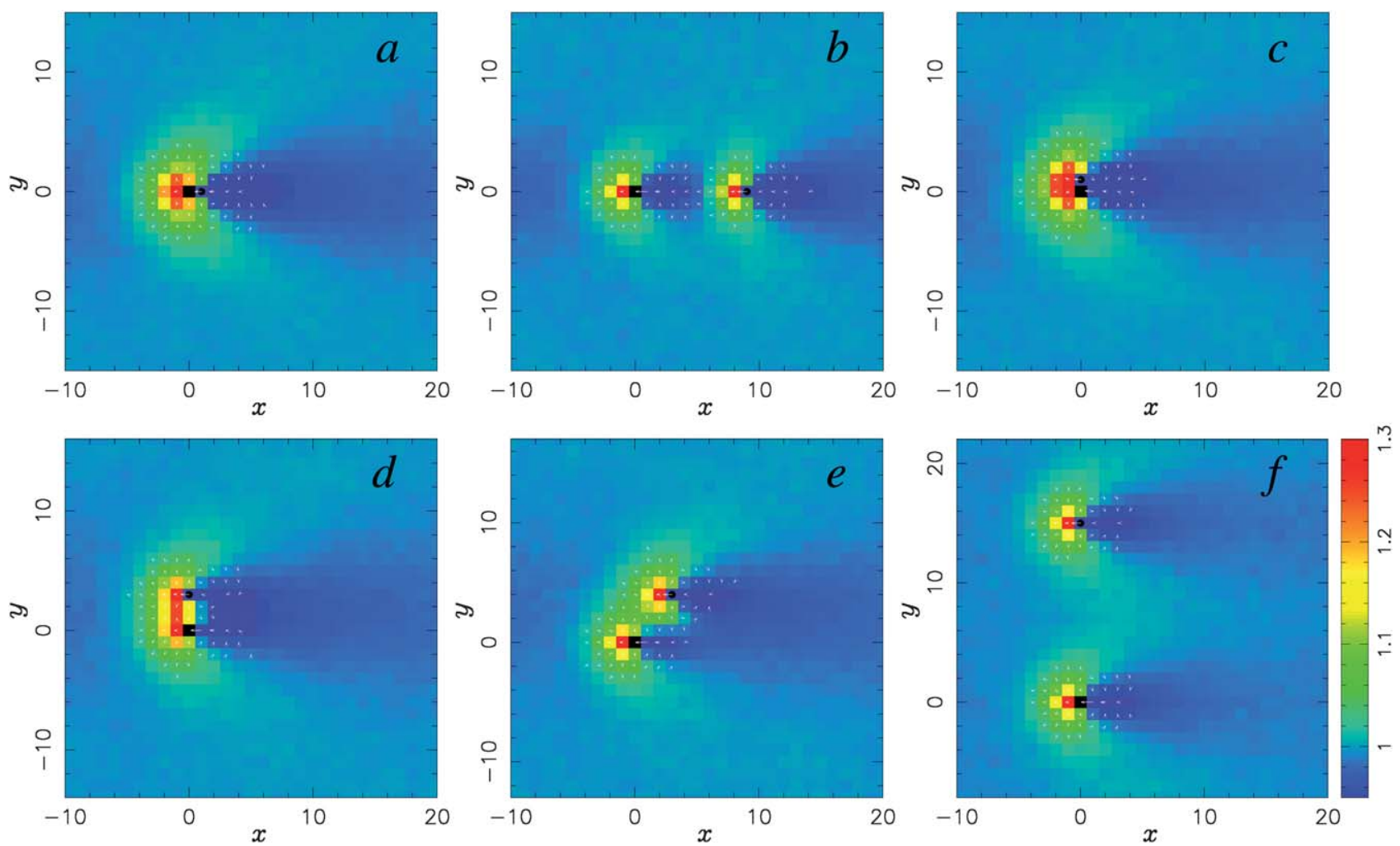

Fig. 8 Microstructural changes produced by two BPs. Colour map of the mean local occupation times of the bath particles $g_{0}(x, y)$, for six different configurations of two BPs, defined in the reference frame of the leading BP (black square), with $\rho=1 / 2$ and $\beta \sigma F=5$. The arrows define the vector velocity field. Velocities with magnitude less than 0.005 are not plotted. 

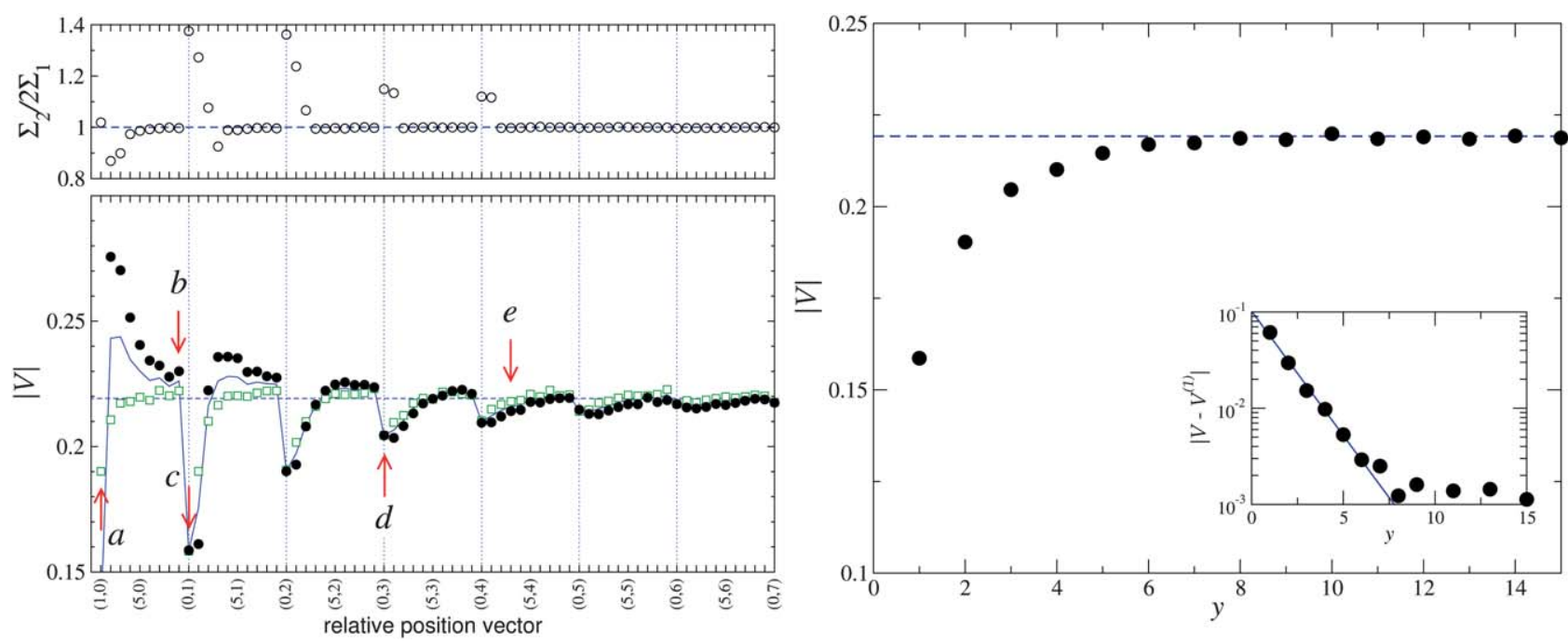

Fig. 9 Drift velocities of the BPs in different configuration Left lower panel: magnitude of the $x$-component of the velocity of the leading (open squares) and trailing (solid circles) BPs for different mutual orientations, $\beta \sigma F=5$ and $\rho=1 / 2$. The blue dashed line defines the velocity $V^{(1)}$ of a single isolated BP, while the solid blue line defines the center-of-mass velocity of a pair of BPs. The arrows indicate some of the configurations depicted in Fig. 8. Left upper panel: the ratio of "effective" inhomogeneities created by two BPs and a single BP (see the text for the explanations). Right panel: drift velocity of the transversal configurations as a function of the separation $y$. The inset shows that the drift velocity converges to $V^{(1)}$ exponentially.

that hinder the motion of the latter reducing the average velocity of the pair. The blocking effect is no longer present in the next configuration $(2,0)$ for which (together with the configuration $(3,0)$ ), the velocity of the center-of-mass is the largest. This signifies that such a pair of the BPs creates the smallest microstructural changes, and in response, encounters the least jamming-induced frictional force, hence the least possible dissipation.

Curiously enough, the velocity of the center-of-mass of such a pair is always higher than the velocity of a single isolated BP. This can be seen in Fig. 3, in which we compare numerical forcevelocity relations for a single BP (solid squares) and a pair in the configuration $(2,0)$ (open circles), for different values of the driving force. The center-of-mass velocity is also higher than the velocity of a single isolated BP for the configurations $(3,0)$, $(4,0)$ to $(9,0),(2,1)$ to $(9,1)$ and $(4,2)$ to $(9,2)$. On the contrary, the microstructural changes of the medium induce the largest frictional force on pairs $(0, y)$, for which the BPs are in perpendicular orientation with respect to the field direction, such as, e.g., configurations (c) and (d). These configurations are indicated in Fig. 9 by vertical arrows. Due to the symmetry of the problem, in these configurations the velocities of the leading and trailing BPs coincide, and are smaller than the velocity of a single isolated BP.

To better understand such a behaviour we study a measure of the size of the inhomogeneity created by the BPs. This configuration-dependent measure that we call $\Sigma$, equals the sum of the mean occupation times of the bath particles, over the lattice sites on which the absolute deviation of $g_{0}(x, y)$ from the mean value $\rho$ exceeds a certain threshold $\mathcal{G}$ :

$$
\Sigma=\sum_{\delta g>\mathcal{G}}\left\langle g_{0}(x, y)\right\rangle,
$$

where $\delta g=\left|\left\langle g_{0}(x, y)\right\rangle-\rho\right|$. To look at the correlation between the size of the inhomogeneity and the jamming-induced interaction among different BPs, we denote $\Sigma_{n}(n=1,2)$, as the inhomogeneity measure (eqn 9), computed for $n$ BPs. In the left top panel of Fig. 9 we present the ratio $\Sigma_{2} / 2 \Sigma_{1}$ for different configurations of the BPs, clearly showing that the largest (smallest) velocities of a pair of BPs occur for smallest (largest) values of $\Sigma_{2} / 2 \Sigma_{1}$. In particular, for the pair configurations whose center-of-mass velocity is larger than the single BP, we obtain $\Sigma_{2} / 2 \Sigma_{1}<1$, indicating that the overall size of the inhomogeneity produced by the pair of BPs in such configurations is smaller than the corresponding size of two isolated BPs.

Finally, in the right panel of Fig. 9 we show that at large separations along the $y$-axis the velocity of the pair approaches the velocity of a single isolated BP exponentially, as is the case of configuration (f), corresponding to the orientation $(0,15)$.

\section{Conclusions}

To recap, we have addressed here a problem of effective, nonequilibrium interactions that emerge between two driven probes in a medium of randomly moving hard-core (but otherwise noninteracting) particles-a quiescent bath. We have shown that for a sufficiently dense medium the probes experience an attractive force towards each other, leading to their statistical pairing. Such a pairing sets in when the non-equilibrium inhomogeneities in the distribution of the bath particles, created by the probes, start to interfere. The inhomogeneities around each driven probe decay exponentially with the distance from the probe, except for the wake of the probe in which the decay is algebraic. Consequently, these non-equilibrium interactions are anisotropic and typically short-ranged, except for the situations when the second driven probe appears in the wake of the first one.

The formation of pairs reduces the overall size of the inhomogeneity, minimizing the frictional drag force the medium exerts on each probe. As a result, in some configurations the center-of-mass of a pair propagates faster than a single isolated 
BP. The jamming-induced forces, which emerge in the situation under study, are very different from fundamental physical interactions, exist only in the presence of an external force, and require the presence of a quiescent bath to mediate the interactions between the driven intruders.

We note that our results have been obtained for a somewhat simplified model of a non-interacting lattice gas with simple exclusion dynamics, which allowed us to single out the effect of the jamming-induced interactions. This model can be generalized in several directions. First of all, one may consider a situation appropriate to a colloidal solution, when some solvent is present. The solvent itself will produce long-range hydrodynamic interactions between the driven probes and correlate dynamics of the bath particles (see Ref. 19 and references therein). One may expect that the pairing effect will become more pronounced in this case. Second, we have considered the case of just two driven probes. It might be interesting to study the specific features of pairing in the situation when there are many of them - the effect observed here seems very much like "an elementary act" for the phenomenon of lane formation in partially driven colloids. ${ }^{22,23}$ Finally, we note that we have focused here solely on the case when the biased motion of the BPs results from the presence of an external force acting on them. For biased motion in intracellular media or under molecular crowding conditions, it might also be interesting to consider other types of biased motion, e.g., the cases of self-propelled particles or swimmers. These important situations merit further investigation.

\section{Acknowledgements}

We thank J. Talbot, J. L. Lebowitz and H. Spohn for helpful discussions. C. M.-M. acknowledges support from the European Research Council and the Academy of Finland. G.O. is partially supported by Agence Nationale de la Recherche (ANR) under grant "DYOPTRI".

\section{References}

1 C. Gutsche, F. Kremer, M. Krüger, M. Rauscher, R. Weeber and J. Harting, J. Chem. Phys., 2008, 129, 084902.

2 R. Candelier and O. Dauchot, Phys. Rev. E: Stat., Nonlinear, Soft Matter Phys., 2010, 81, 011304.

3 M. Krüger and M. Rauscher, J. Chem. Phys., 2009, 131, 094902.

4 C. Reichhardt and C. J. O. Reichhardt, Phys. Rev. Lett., 2004, 92, 108301.

5 S. F. Burlatsky, G. Oshanin, A. Mogutov and M. Moreau, Phys. Lett. A, 1992, 166, 230.

6 S. F. Burlatsky, G. Oshanin, M. Moreau and W. P. Reinhardt, Phys. Rev. E: Stat. Phys., Plasmas, Fluids, Relat. Interdiscip. Top., 1996, 54, 3165.

7 G. Oshanin, O. Bénichou, S. F. Burlatsky and M. Moreau, Instabilities and nonequilibrium structures IX, Springer, Dordrech, Netherlands, 2004, pp. 33-74.

8 J. De Coninck, G. Oshanin and M. Moreau, Europhys. Lett., 1997, 38, 527.

9 O. Bénichou, A. M. Cazabat, J. De Coninck, M. Moreau and G. Oshanin, Phys. Rev. Lett., 2000, 84, 511.

10 O. Bénichou, A. M. Cazabat, J. De Coninck, M. Moreau and G. Oshanin, Phys. Rev. B: Condens. Matter Mater. Phys., 2001, 63, 235413.

11 O. Bénichou, J. Klafter, M. Moreau and G. Oshanin, Phys. Rev. E: Stat. Phys., Plasmas, Fluids, Relat. Interdiscip. Top., 2000, 62, 3327.

12 T. M. Squires and J. F. Brady, Phys. Fluids, 2005, 17, 073101.

13 A. S. Khair and J. F. Brady, J. Fluid Mech., 2006, 557, 73.

14 J. Dzubiella, H. Löwen and C. N. Likos, Phys. Rev. Lett., 2003, 91, 248301.

15 M. Krüger and M. Rauscher, J. Chem. Phys., 2007, 127, 034905.

16 A. S. Khair and J. F. Brady, Proc. R. Soc. London, Ser. A, 2007, 463, 223.

17 H. Spohn, Large Scale Dynamics of Interacting Particles, Springer, New York, 1991.

18 K. Binder, Monte Carlo methods in statistical physics, Springer, Berlin, 1979.

19 M. Janosi, T. Tél, D. E. Wolf and J. A. C. Gallas, Phys. Rev. E: Stat. Phys., Plasmas, Fluids, Relat. Interdiscip. Top., 1997, 56, 2858.

20 L. van Hove, Phys. Rev., 1954, 95, 249.

21 O. Bénichou and G. Oshanin, Phys. Rev. E: Stat., Nonlinear, Soft Matter Phys., 2002, 66, 031101.

22 Dzubiella, G. P. Hoffmann and H. Löwen, Phys. Rev. E: Stat., Nonlinear, Soft Matter Phys., 2002, 65, 021402.

23 M. Rex and H. Löwen, Phys. Rev. E: Stat., Nonlinear, Soft Matter Phys., 2007, 75, 051402. 